\title{
ESTUDO COMPARATIVO ENTRE OS MÉTODOS GRADIENTE REDUZIDO GENERALIZADO E ALGORITMO GENÉTICO EM OTIMIZAÇÃO COM MÚLTIPLAS RESPOSTAS
}

\section{COMPARATIVE STUDY BETWEEN THE GENERALIZED REDUCED GRADIENT AND GENETIC ALGORITHM IN MULTIPLE RESPONSE OPTIMIZATION}

\author{
Fabrício Maciel Gomes*, ${ }^{* *}$ E-mail: fmgomes@usp.br \\ Félix Monteiro Pereira* E-mail: felixmpereira@usp.br \\ Fernando Augusto Silva Marins* E-mail: fmarins@feg.unesp.br \\ Messias Borges Silva, ${ }^{*}$ E-mail: messias@dequi.eel.usp.br \\ * Universidade de São Paulo (USP), Lorena, SP \\ ** Universidade Estadual Paulista (UNESP), Guaratinguetá, SP
}

\begin{abstract}
Resumo: Neste trabalho foi realizado um estudo comparativo entre as metodologias de otimização Gradiente Reduzido Generalizado (GRG) e Algoritmo Genético (AG) para a otimização de processos com múltiplas respostas. Para estimar os parâmetros que minimizam a função objetivo foram utilizadas respostas geradas por planejamento de experimentos de forma aglutinada, as quais foram incorporadas à função objetivo. Os estudos de caso utilizados foram baseados em trabalhos selecionados na literatura e, para cada experimento selecionado, foi realizada a otimização dos valores dos parâmetros do processo utilizando as duas metodologias, o GRG, por meio de uma planilha do Microsoft Excel e o AG utilizando o software Scilab. Foram realizadas 10 replicações e calculada a média dos resultados obtidos. A comparação entre os métodos foi realizada com base em medidas de desempenho, por meio da distância média percentual. O AG apresentou melhores resultados em comparação com o GRG.
\end{abstract}

Palavras-chave: Otimização. Múltiplas Respostas. Algoritmo Genético. GRG.

Abstract: In this paper we present a comparative study between the Generalized Reduced Gradient (GRG) and Genetic Algorithm (GA) methods to optimize multiple-response processes. Results from experiment design were used to compose the objective function to be minimized. The case studies in this work were selected from literature. A Microsoft Excel spreadsheet was used for parameters optimization using GRG, and the Scilab software was used to GA. Ten replicates were performed and the mean of the results was obtained. To assess the methods was used performance measures based on the mean percentage error. From the performance measures used, the AG showed better results compared to the GRG, indicating that the AG can generate better responses than GRG.

Keywords: Optimization. Multiple Response. Genetic Algorithm. GRG.

\section{INTRODUÇÃO}

As variações de demanda dos clientes e os avanços tecnológicos tornam complexa a determinação de uma melhoria de processo, pois, em geral, é necessário considerar várias respostas a fim de se atingir uma melhoria global no 
processo. Dessa forma, a melhoria simultânea de múltiplas respostas é uma das prioridades em vários ramos industriais (TSAl et al., 2010).

A melhoria de um processo consiste na busca pelas melhores condições de ajuste para uma operação ou um conjunto de operações. O processo de busca pode partir de uma solução inicial ou de um conjunto de soluções iniciais, realizar melhoramentos progressivos até chegar a outro conjunto que contenha uma ou todas as melhores soluções possíveis.

Os problemas utilizados na melhoria de processos podem ser formulados genericamente como mostrado em (1).

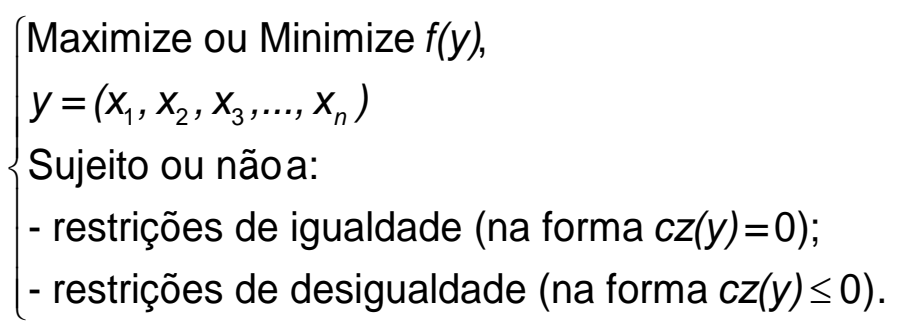

A melhoria de um processo visa geralmente a redução de custos ou o incremento de ganhos pela determinação de condições pertencentes a um conjunto de variáveis $y\left(x_{1}, x_{2}, x_{3}, \ldots, x_{n}\right)$ capaz de gerar melhores valores da função $f(y)$, considerando as restrições impostas.

Um modelo matemático capaz de descrever o comportamento de um processo pode ser obtido por técnicas determinísticas ou estocásticas. Uma ferramenta útil em estudos de otimização com múltiplas respostas é a metodologia de superfície de resposta em sua versão multivariada (DÍAS-GARCÍA; BASHIRI, 2014). Com essa abordagem é possível determinar uma relação analítica entre a resposta e as variáveis independentes utilizando uma quantidade menor de pontos experimentais (KHURI; CORNELL, 1987).

Dependendo da forma de obtenção da função objetivo e/ou restrições, o problema pode ser linear ou não linear. Além disso, as variáveis que compõem a função objetivo podem assumir valores reais, inteiros ou ambos.

Em problemas de minimização, uma solução $y^{*}$ é chamada mínima global quando não existir outra $y$, dentro do espaço de busca, que forneça um valor para a função objetivo $f(y)$ menor do que $f\left(y^{*}\right)$. Em problemas de maximização, o máximo 
global $y^{*}$ atende a condição $f\left(y^{*}\right)$ maior do que $f(y)$ para qualquer $x$ pertencente ao espaço de busca, como pode ser observado na Figura 1.

Figura 1 - Exemplos de ponto de máximo e de mínimo em uma função

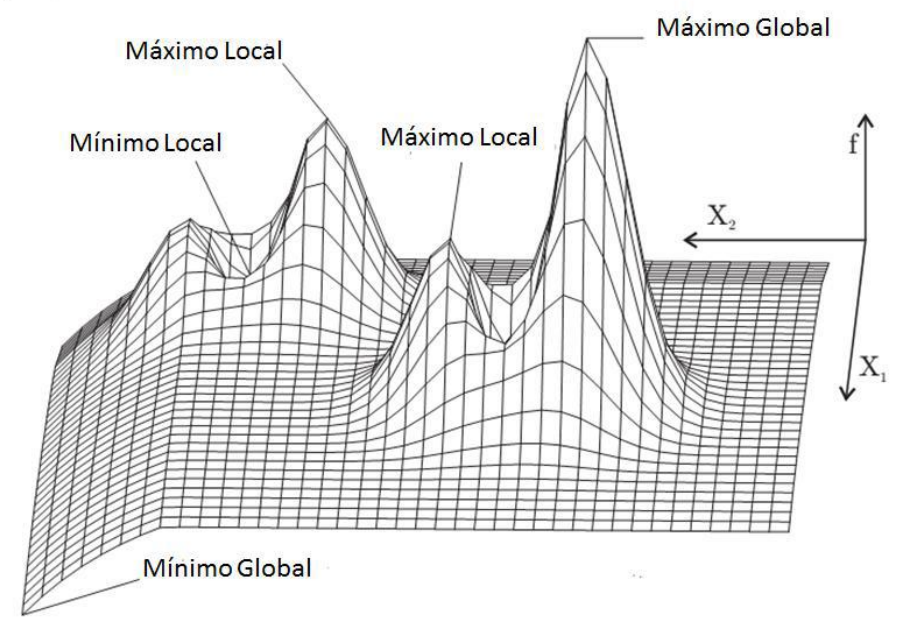

Fonte: adaptado de Weise (2009)

Quando uma solução $y$ possui $f(y)$ mínimo apenas dentro de uma determinada região próxima a $y$, chamada de vizinhança de $y$, diz-se que o mínimo é local. Mínimos locais podem vir a ser boas soluções, porém não são as melhores. Dependendo do método de busca utilizado, esses pontos podem ser indesejáveis por interromperem a busca por melhores soluções.

Para contornar este tipo de problema pode-se utilizar algoritmos heurísticos, como o método do Gradiente Reduzido Generalizado (GRG) que, mesmo não sendo capazes de garantir a obtenção do mínimo global, podem oferecer soluções aceitáveis para problemas complexos com baixo custo computacional (IGNíZIO; CAVALIER, 1994). Outra forma eficaz é a utilização de meta-heurísticas, como os algoritmos genéticos que propiciam a busca por pontos ótimos sem a necessidade de calcular todas as soluções possíveis empregando conceitos de evolução Darwiniana (HAMMOUCHE et al., 2010).

\section{FUNDAMENTAÇÃO TEÓRICA}

Esta seção tem como objetivo apresentar, de maneira resumida, as técnicas utilizadas neste trabalho. 


\subsection{Otimização}

A busca de um estado ideal é um dos princípios fundamentais da natureza. Essa busca se inicia no microcosmo onde os átomos formam ligações que minimizam a energia de seus elétrons (PAULING, 1960). As moléculas formam corpos sólidos durante o processo de congelamento, tendendo a assumir estruturas cristalinas com um estado de mínima energia. Estes processos resultam puramente das leis da física.

Na história humana, a incessante busca por melhorias das condições de vida pode também ser entendida como um processo de otimização.

Nas empresas, a produção deve ser otimizada a fim de se obter produtividade e lucros máximos mantendo os custos em um mínimo possível.

Um processo de otimização nem sempre implica na determinação das condições ótimas de operação, pois, em muitos casos, é impossível estabelecer uma condição ótima de operação devido a uma quantidade ilimitada de variáveis que impactam no processo. Nesses casos, o que se pode determinar são as condições de melhoria por meio da seleção de pontos máximos dentro de um espaço de busca pré-determinado (DEHURI; CHO, 2009).

\subsection{Otimização de Múltiplas Respostas}

Normalmente, os processos industriais e os produtos por eles gerados possuem características de qualidade diversas, algumas mais importantes que outras na proporção do valor que o cliente atribui a cada uma delas. Uma indústria pode ter, dentre outras, as seguintes respostas sujeitas a otimização:
a) Minimizar o lead time;
b) Maximizar a produtividade;
c) Minimizar os custos de produção;
d) Maximizar a qualidade de seu produto;
e) Minimizar o impacto com o meio ambiente.

As respostas "d" e "e" são comumente conflitantes com a minimização de 
custos de produção. Os conflitos entre as respostas nem sempre são óbvios e podem gerar uma série de complicações durante o processo de otimização (WEISE, 2009).

Na otimização de múltiplas respostas normalmente não há nenhuma solução que seja ideal para todos os objetivos impostos. Dessa forma, qualquer solução pode ser sempre melhorada em pelo menos um objetivo (BAZGAN et al., 2015). Portanto, pode-se considerar que o resultado de uma otimização de múltiplas respostas gere um conjunto de soluções ótimas, conhecido como soluções nãodominadas.

Soluções não-dominadas são também chamadas de soluções Pareto-ótimas. Todas as soluções que são Pareto-ótimas constituem o conjunto Pareto. Os valores objetivos do conjunto de Pareto no espaço objetivo constituem a fronteira de Pareto (SUDENG; WATTANAPONGSAKORN, 2015).

A resolução de problemas multiobjetivo é dividida, basicamente, em duas etapas:

a) Determinação de soluções eficientes;

b) Etapa de decisão.

O primeiro aspecto diz respeito à determinação do conjunto de soluções Pareto-ótimas dentro da região viável do conjunto de respostas. O segundo aspecto se refere à determinação da melhor resposta dentre as relacionadas pelo conjunto Pareto-ótimo. Este procedimento também é conhecido como decisor.

Segundo Veldhuizen e Lamont (2000), os métodos de resolução de problemas com múltiplas respostas podem ser classificados em três categorias distintas, de acordo com a combinação realizada entre o método de otimização e a etapa de decisão:

a) Decisão antes do processo de procura: quando o decisor atribui os pesos para cada objetivo que compõe o problema;

b) Decisão durante o processo de procura: é o procedimento que faz as escolhas durante o processo de obtenção das soluções;

c) Decisão após o processo de procura: quando a determinação da melhor solução é realizada após a determinação das soluções eficientes. 
A apresentação das decisões após a etapa de definição das soluções eficientes é a mais lógica das três, isto porque as escolhas serão feitas de acordo com as respostas finais encontradas. Ou seja, como já dito, com o conjunto Paretoótimo definido torna-se possível conhecer o comportamento do problema em relação aos objetivos analisados. Conhecendo-se as relações de dependência entre eles, a escolha final é facilitada (AVILA, 2006).

\subsection{Planejamento de Experimentos}

O planejamento de experimentos consiste em um conjunto de ensaios estabelecidos sob critérios estatísticos e científicos, com a finalidade de determinar a influência de diversas variáveis sobre determinadas respostas (TORRES JÚNIOR; QUININO, 2014).

$\mathrm{O}$ arranjo experimental mais comum é o fatorial completo para o qual o número de experimentos é igual ao número de níveis experimentais elevado ao número de fatores. Montgomery e Runger (2012) afirmam que "planejamentos fatoriais" são frequentemente usados nos experimentos envolvendo vários fatores e que "experimentos fatoriais" são a única maneira de descobrir interações entre variáveis de processo.

Segundo Haridy et al. (2011), o Planejamento de Experimentos é um método estruturado e organizado, utilizado na determinação do relacionamento de diferentes fatores de entrada e saída do processo, envolvendo a definição do conjunto de experimentos, nos quais todos os fatores relevantes são variados sistematicamente.

Por meio da análise dos resultados obtidos pode-se determinar o grau de influência na variável resposta de cada fator utilizado, assim como as interações entre os fatores e as condições ótimas.

Segundo Melo et al. (2013), o método do planejamento de experimentos utilizado para otimização experimental proporciona resultados importantes que auxiliam o engenheiro de produção ou de processo, na análise de diagnóstico do processo de manufatura. 


\subsection{Distância Percentual Média (DPM)}

A função aglutinadora Distância Percentual Média (DPM), pode ser obtida como uma função das variáveis de resposta $\left(\hat{y}_{i}\right)$ e valores alvo $\left(T_{i}\right)$ individuais, como apresentado em (2), onde $p$ é o número de parâmetros $\left(x_{i}\right)$ a serem otimizados (GOMES et al., 2015).

$$
D P M=\frac{\sum_{i=1}^{p} \frac{\left|\hat{y}_{i}-T_{i}\right|}{T_{i}}}{p}
$$

Segundo Kim e Lin (2006), este é o método que tem obtido melhores resultados quando aplicados na avaliação e na comparação dos métodos de otimização.

\subsection{Algoritmo Genético (AG)}

A meta-heurística Algoritmo Genético (AG), introduzida por Holland em 1975, faz parte de um conjunto de algoritmos mais abrangente, chamados de Algoritmos Evolutivos. Nas décadas de 60 e 70 vários pesquisadores de diferentes pontos dos Estados Unidos e da Europa convergiam para a ideia de mimetizar o mecanismo de evolução biológica a fim de resolver problemas de diversas áreas, em especial o de otimização. Dessas pesquisas resultaram diferentes abordagens e algoritmos, sendo os principais representantes as estratégias evolutivas (RECHENBERG, 1973), a programação evolutiva (FOGEL et al., 1966) e os algoritmos genéticos (HOLLAND, 1975; GOLDBERG, 1989).

Segundo Cheng et al. (2002), o AG demonstra um bom desempenho quando utilizado para otimização de problemas com múltiplas respostas aglutinadas.

Para compreender o funcionamento dos algoritmos genéticos é necessário realizar uma analogia com a teoria da evolução das espécies. Desse modo, o funcionamento do AG possui as seguintes características: 
a) Inicialmente é gerada uma população formada por um conjunto aleatório de indivíduos, que podem ser vistos como possíveis soluções do problema;

b) Durante o processo evolutivo, esta população é avaliada, sendo que para cada indivíduo é atribuída uma nota, ou índice, que reflete sua habilidade de adaptação a determinado ambiente;

c) Uma porcentagem dos indivíduos mais adaptados é mantida, enquanto os outros são descartados;

d) Os membros mantidos pela seleção podem sofrer modificações em suas características fundamentais por meio de cruzamentos, mutações ou recombinação genética gerando descendentes para a próxima geração;

e) Este processo, chamado de reprodução, é repetido até que uma solução satisfatória seja encontrada. Embora possam parecer simplistas do ponto de vista biológico, estes algoritmos são suficientemente complexos para fornecer mecanismos de busca adaptativos poderosos e robustos.

O Quadro 1 apresenta um pseudocódigo de um AG clássico.

Quadro 1 - Pseudocódigo da meta-heurística AG
Algoritmo AG $\left(\mu, p_{c}, p_{m}\right)$
Gere a população inicial de tamanho $\mu$
enquanto (critério de parada não for satisfeito) faça
selecione a população de pais
selecione o operador de recombinação com probabilidade $p_{c}$
selecione o operador de mutação com probabilidade $p_{m}$
avalie a população de filhos
selecione a nova população
fim-enquanto
fim-algoritmo

Fonte: adaptado de Reeves (2003)

\subsubsection{Funcionamento de um AG}

Para realizar transformações em uma população, os operadores genéticos atuam de maneira que, a cada nova geração, os novos indivíduos sejam mais capazes de se adaptar ao meio. Assim, os operadores genéticos contribuem para que o processo de evolução ocorra de fato.

As etapas de um AG são as apresentadas a seguir, como descrito por Mendes (2013): 


\section{a) Inicialização}

Um AG tem como primeira etapa a criação de uma população inicial que é realizada de maneira aleatória, a esta função dá-se o nome de inicialização.

A geração de cromossomos que compõem a população inicial tem como objetivo aumentar a diversidade genética da população inicial garantindo um maior alcance no espaço de busca.

Caso a inicialização da população não ocorra de forma randômica, esta poderá convergir prematuramente significando que, em um curto espaço de tempo, a população possuirá indivíduos muito semelhantes, ou seja, com pouca diversidade genética, o que dificultará na escolha da melhor solução possível para o problema estudado.

Após a criação da população inicial, é necessário avaliar todos os cromossomos gerados quanto a sua aptidão ao meio.

\section{b) Função de Aptidão}

Pela teoria da seleção natural, a aptidão é exercida pelo meio em que o indivíduo vive. Quando um determinado indivíduo não se encontra apto para sobreviver em um determinado meio, ele tem menor probabilidade de se recombinar (crossover) e assim não transmitir a sua carga genética para as próximas gerações.

No $A G$, é desejável que a função aptidão mantenha os melhores resultados de uma geração permitindo que os mesmos sejam replicados para a próxima geração. Assim, ao gerar uma nova população, os melhores genomas "poderão ser autorizados" a serem transmitidos de forma inalterada para a população futura. Este método é conhecido como seleção elitista e esses genomas selecionados são chamados de membros de elite da população mais antiga (FAGHIHI et al., 2014).

\section{c) Seleção}

A seleção é executada após a determinação da aptidão dos cromossomos gerados. Este processo está baseado no fato de que indivíduos mais capazes de sobreviver ao meio têm maior probabilidade de gerar descendentes, enquanto que 
indivíduos menos capazes têm menor probabilidade de gerar descendentes. Portanto a operação de seleção não pode ser realizada de forma que somente o indivíduo mais capaz possa gerar descendentes, pois existe a chance de um indivíduo menos capaz contribuir com sua carga genética com as populações futuras.

\section{d) Cruzamento}

O cruzamento (crossover ou recombinação) é efetuado logo após a seleção. Nesta operação ocorre a troca de segmentos entre pares de cromossomos selecionados dando origem a novos indivíduos que formarão a população da geração seguinte.

O propósito da operação de cruzamento é repassar características dos indivíduos mais aptos da população por meio da troca de segmentos de informações entre si, originando novos indivíduos.

Os métodos mais comuns de cruzamento nos Algoritmos Genéticos são os de ponto único, de ponto duplo e de pontos aleatórios:

- Método de ponto único: um ponto de corte aleatório é escolhido, o material genético dos pais é trocado dando origem a dois novos cromossomos formados pela combinação das características genéticas dos pais, como apresentado na Figura 2.

Figura 2 - Cruzamento em ponto único



Fonte: adaptado de Mendes (2013)

- Método de ponto duplo: dois pontos de cruzamento aleatórios são escolhidos ocorrendo a troca entre os materiais genéticos dos pais de forma intercalada, como mostrado na Figura 3. 
Figura 3 - Cruzamento em ponto duplo

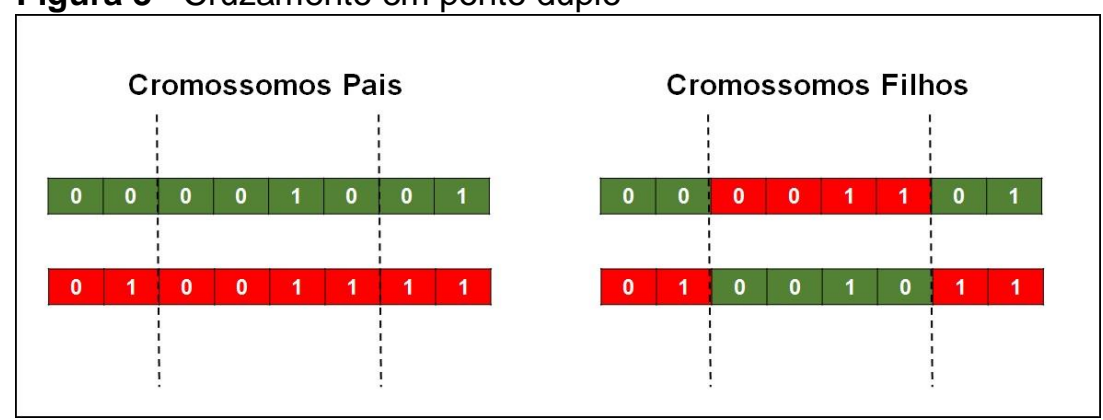

Fonte: adaptado de Mendes (2013)

- Método de pontos aleatórios: utiliza como base máscaras (cromossomos binários) onde, no local em que os alelos possuem o valor "1", utiliza-se inicialmente o material genético de um dos pais e, no local em que os alelos possuem o valor "0", o material do outro pai. Essa máscara pode ser invertida dando início a um novo processo de cruzamento, como mostrado na Figura 4.

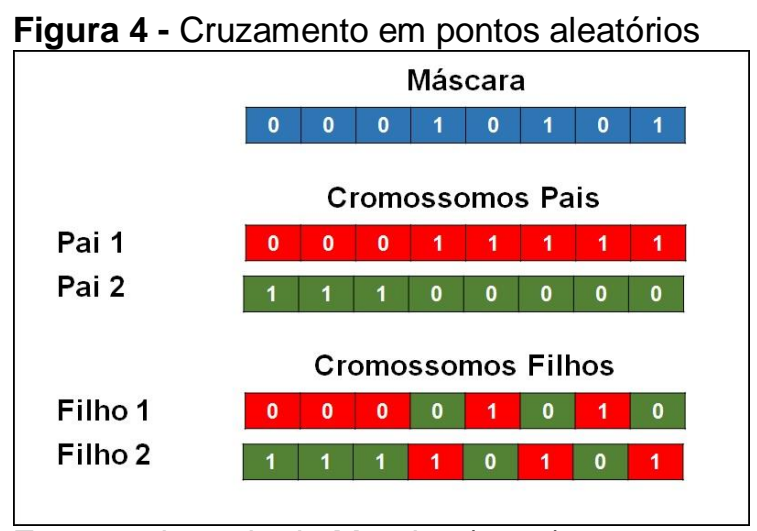

Fonte: adaptado de Mendes (2013)

\section{e) Mutação}

A mutação é realizada após o cruzamento e tem o objetivo de realizar modificações aleatórias em determinadas propriedades genéticas de uma população. Esta operação possibilita a obtenção de propriedades genéticas que não existiam ou que eram pouco encontradas na população atual.

A mutação permite a introdução e manutenção da diversidade genética da população. Portanto, a execução do operador de mutação possibilita garantir que o espaço de busca possivelmente não será igual a zero. 
A taxa de mutação deve acometer uma pequena parcela da população, pois, caso essa taxa seja elevada, os indivíduos gerados pouco se assemelharão aos seus pais.

Existem várias técnicas de mutação, dentre elas: mutação aleatória onde um valor é sorteado para substituir o que sofrerá a mutação como mostrado na Figura 5; mutação por troca, onde $n$ pares de genes são sorteados e após o sorteio os pares trocam valores entre si, como apresentado na Figura 6.

Figura 5 - Mutação aleatória.

\begin{tabular}{|l|l|l|l|l|l|l|l|l|l|l|l|l|l|l|l|}
\hline \multicolumn{1}{|c|}{ Cromossomos antes da mutação } & \multicolumn{1}{c|}{ Cromossomos depois da mutação } \\
\hline 0 & 0 & 0 & 1 & 0 & 1 & 0 & 1 \\
\hline
\end{tabular}

Fonte: adaptado de Mendes (2013)

Figura 6 - Mutação por troca

\begin{tabular}{|l|l|l|l|l|l|l|l|l|l|l|l|l|l|l|l|}
\hline \multicolumn{1}{c|}{ Cromossomos antes da mutação } & \multicolumn{1}{c|}{ Cromos depois da mutação } \\
\hline 0 & 0 & 0 & 1 & 0 & 1 & 0 & 1 \\
\hline
\end{tabular}

Fonte: adaptado de Mendes (2013)

\section{f) Atualização}

A atualização consiste na substituição da população antiga por uma nova, formada pelo cruzamento dos indivíduos selecionados da população anterior. As formas mais conhecidas de atualização são: a estratégia soma, onde alguns indivíduos da população anterior convivem com a população formada por seus filhos (elitista); a estratégia vírgula, na qual a população anterior não convive com a próxima população, perdendo-se então soluções boas encontradas.

\section{g) Finalização}

O operador de finalização determina se a execução do Algoritmo Genético será concluída ou não, com base em uma condição de parada previamente estabelecida. A condição de parada pode ser a quantidade gerações desenvolvidas ou o grau de proximidade dos valores de aptidão de cada cromossomo da população. 


\subsection{Gradiente Reduzido Generalizado}

O método do Gradiente Reduzido Generalizado (GRG) foi apresentado por Abadie e Carpentier (1969) para solucionar problemas de programação matemática com uma função objetivo não linear e restrições lineares. Este trabalho foi baseado no método do Gradiente descrito por Rosen (1960) e no método do Gradiente Reduzido descrito por Wolfe (1963).

Waren e Lasdon (1979) deram uma descrição detalhada do desempenho de vários códigos computacionais, em uso na época, informando a qualidade diferenciada do GRG. Posteriormente, Lasdon et al. (1980) efetuaram algumas modificações no algoritmo e o batizaram de GRG2. Ressalta-se que estas modificações foram realizadas no algoritmo computacional preservando-se o método original.

Segundo Köksoy e Yalcinoz (2006), o GRG é o método mais empregado na resolução de problemas com múltiplas respostas, fato este devido a sua facilidade de implantação e de seu algoritmo estar disponibilizado no pacote Solver que acompanha o software Microsoft Exce|®. Entretanto, a maior limitação da utilização do GRG provém do fato de que é necessário que a estimativa inicial dos parâmetros seja próxima ao valor do ponto ótimo para que se tenha uma garantia de convergência para o ponto ótimo.

\section{MÉTODOS}

O presente estudo tem como objetivo realizar uma avaliação comparativa entre o desempenho da meta-heurística AG e da heurística GRG. Como base de comparação para os métodos propostos foi utilizada a DPM como método de aglutinação.

Para se proceder com este estudo, foram selecionados cinco trabalhos na literatura que envolvem a solução de problemas com múltiplas respostas. Neste trabalho, foram realizadas 10 replicações em cada um deles e calculada a média e o desvio-padrão dos resultados obtidos.

Os casos selecionados na literatura para a execução deste estudo são apresentados a seguir. 


\subsection{Estudos de Casos}

Os métodos aqui apresentados (AG e GRG) foram aplicados a 4 estudos de casos descritos a seguir.

\subsubsection{Caso 1 - Derringer e Suich (1980)}

O problema descrito por Derringer e Suich (1980), consiste na otimização de quatro índices de qualidade de pneus $\left(\hat{y}_{i}\right)$ associados a três fatores de controle ou variáveis de decisão $\left(x_{i}\right): \hat{y} 1$, índice de abrasão; $\hat{y} 2$, módulo $200 \%$; $\hat{y}_{3}$, alongamento de ruptura; $\hat{y} 4$, dureza; $x_{1}$, nível de sílica hidratada; $x_{2}$, nível de silano com agente ligante e $x_{3}$, concentração de enxofre. As experiências foram realizadas com base num desenho de compósito central com seis pontos centrais e as equações de regressão das respostas de segundo grau obtidas são apresentadas em 3-6.

$$
\begin{aligned}
& \hat{y}_{1}=139,12+16,49 x_{1}+17,88 x_{2}+10,91 x_{3}-4,01 x_{1}^{2}-3,45 x_{2}^{2}-1,57 x_{3}^{2}+5,13 x_{1} x_{2} \\
& +7,13 x_{1} x_{3}+7,88 x_{2} x_{3} \\
& \hat{y}_{2}=1261,11+268,15 x_{1}+246,50 x_{2}+139,48 x_{3}-83,55 x_{1}^{2}-124,79 x_{2}^{2}+199,17 x_{3}^{2} \\
& +68,38 x_{1} x_{2}+94,13 x_{1} x_{3}+104,38 x_{2} x_{3} \\
& \hat{y}_{3}=400,38-99,67 x_{1}-31,40 x_{2}+73,92 x_{3}+7,93 x_{1}^{2}+17,31 x_{2}^{2}+0,43 x_{3}^{2}+8,75 x_{1} x_{2} \\
& +6,25 x_{1} x_{3}+1,25 x_{2} x_{3} \\
& \quad \hat{y}_{4}=68,91-1,41 x_{1}+4,32 x_{2}+1,63 x_{3}-1,56 x_{1}^{2}+0,06 x_{2}^{2}-0,32 x_{3}^{2}-1,63 x_{1} x_{2} \\
& \quad+0,13 x_{1} x_{3}-0,25 x_{2} x_{3}
\end{aligned}
$$

Os alvos $\left(T_{i}\right)$ para a otimização de cada resposta foram determinados pelos autores do referido trabalho e são apresentados nas igualdades apresentadas em 7 .

$$
T_{1}=170 ; T_{2}=1300 ; T_{3}=500 ; T_{4}=67,5
$$

As restrições para este problema estão descritas em 8. 


$$
\begin{aligned}
& -1,663 \leq x_{1} \leq 1,663 ;-1,663 \leq x_{2} \leq 1,663 ;-1,663 \leq x_{3} \leq 1,663 ; \hat{y}_{1} \geq 120 ; \\
& \hat{y}_{2} \geq 1000 ; 400 \leq \hat{y}_{3} \leq 600 ; 65 \leq \hat{y}_{4} \leq 70
\end{aligned}
$$

A função objetivo, resultante da aglutinação das respostas para este estudo de caso está descrito em 9.

$$
D P M=\frac{\left|\frac{\hat{y}_{1}-170}{170}\right|+\left|\frac{\hat{y}_{2}-1300}{1300}\right|+\left|\frac{\hat{y}_{3}-500}{500}\right|+\left|\frac{\hat{y}_{4}-67,5}{67,5}\right|}{4} \cdot 100
$$

\subsubsection{Caso 2: Khuri e Conlon (1981)}

O problema descrito por Khuri e Conlon (1981) tem como objetivo otimizar quatro respostas distintas: $\hat{y}_{1}$, dureza; $\hat{y}_{2}$, coesividade; $\hat{y}_{1}$, elasticidade; $\hat{y}_{3}$, compressão à água. Foram consideradas duas variáveis de decisão: $x_{1}$, cisteína; $x_{2}$, clorato de cálcio. As equações de regressão das respostas de segundo grau obtidas são apresentadas em 10-13.

$$
\begin{aligned}
& \hat{y}_{1}=1,526-0,575 x_{1}-0,524 x_{2}-0,171 x_{1}^{2}-0,098 x_{2}^{2}+0,318 x_{1} x_{2} \\
& \hat{y}_{2}=0,660-0,092 x_{1}-0,010 x_{2}-0,096 x_{1}^{2}-0,058 x_{2}^{2}-0,070 x_{1} x_{2} \\
& \hat{y}_{3}=1,776-0,250 x_{1}-0,078 x_{2}-0,156 x_{1}^{2}-0,079 x_{2}^{2}-0,010 x_{1} x_{2} \\
& \hat{y}_{4}=0,468+0,131 x_{1}+0,073 x_{2}+0,026 x_{1}^{2}+0,024 x_{2}^{2}-0,083 x_{1} x_{2}
\end{aligned}
$$

Os alvos para a otimização de cada resposta são apresentados em 14.

$$
T_{1}=2,68 ; T_{2}=0,69 ; T_{3}=1,90 ; T_{4}=0,71
$$

As restrições para este problema estão descritas em 15.

$$
\begin{aligned}
& -1,414 \leq x_{1} \leq 1,414 ;-1,414 \leq x_{2} \leq 1,414 ; \hat{y}_{1} \geq 0,37 ; \hat{y}_{2} \geq 0,30 \\
& \hat{y}_{3} \geq 1,10 ; \hat{y}_{4} \geq 0,23
\end{aligned}
$$


A função objetivo, resultante da aglutinação das respostas para este estudo de caso está descrito em 16.

$$
D P M=\frac{\left|\frac{\hat{y}_{1}-2,68}{2,68}\right|+\left|\frac{\hat{y}_{2}-0,69}{0,69}\right|+\left|\frac{\hat{y}_{3}-1,90}{1,90}\right|+\left|\frac{\hat{y}_{4}-0,71}{0,71}\right|}{4} \cdot 100
$$

\subsubsection{Caso 3: Vining (1998)}

O problema descrito por Vining (1998) tem como objetivo otimizar duas respostas distintas: $\hat{y}_{1}$, máxima conversão; $\hat{y}_{2}$, atividade térmica. Foram consideradas três variáveis de decisão: $x_{1}$, tempo de reação; $x_{2}$, temperatura de reação; $x_{3}$, quantidade de catalisador. Em 17 e 18, são apresentados os modelos obtidos por regressão.

$$
\begin{gathered}
\hat{y}_{1}=81,09+1,03 x_{1}+4,04 x_{2}+6,20 x_{3}-1,83 x_{1}^{2}+2,94 x_{2}^{2}-5,19 x_{3}^{2}+2,13 x_{1} x_{2} \\
+11,37 x_{1} x_{3}-3,87 x_{2} x_{3} \\
\hat{y}_{2}=60,23+4,26 x_{1}+2,23 x_{3}
\end{gathered}
$$

Os alvos para a otimização das respostas são apresentados em 19.

$$
T_{1}=100 ; T_{2}=57,5
$$

As restrições para este problema são apresentadas em 20.

$$
\begin{aligned}
& -1,663 \leq x_{1} \leq 1,663 ;-1,663 \leq x_{2} \leq 1,663 ;-1,663 \leq x_{3} \leq 1,663 ; \\
& \hat{y}_{1} \geq 80 ; 55 \leq \hat{y}_{2} \leq 60
\end{aligned}
$$

A função objetivo, resultante da aglutinação das respostas para este estudo de caso está descrita em 21.

$$
D P M=\frac{\left|\frac{\hat{y}_{1}-100}{100}\right|+\left|\frac{\hat{y}_{2}-57,5}{57,5}\right|}{2} \cdot 100
$$




\subsubsection{Caso 4 - Castillo et al. (1996)}

O problema apresentado por Castillo et al. (1996), consiste na otimização de seis índices de qualidade: $\hat{y}_{1}$, temperatura máxima na posição $A ; \hat{y}_{2}$, temperatura inicial da ligação na posição $A ; \hat{y}_{3}$, temperatura final da ligação na posição $A ; \hat{y}_{4}$, temperatura máxima na posição $B ; \hat{y} 5$, temperatura inicial da ligação na posição $B$; $\hat{y} 6$, temperatura final da ligação na posição $B$. Foram utilizadas três variáveis de decisão: $x_{1}$, taxa de fluxo de nitrogênio; $x_{2}$, temperatura do nitrogênio; $x_{3}$, temperatura do bloco de aquecimento. Em 22-27, são apresentados os modelos obtidos por regressão.

$$
\begin{gathered}
\hat{y}_{1}=174,93+23,38 x_{2}+3,62 x_{3}-19,00 x_{2} x_{3} \\
\hat{y}_{2}=141,00+6,00 x_{1}+21,02 x_{2}+14,12 x_{3} \\
\hat{y}_{3}=139,53+7,25 x_{1}+16,00 x_{2}+19,75 x_{3} \\
\hat{y}_{4}=154,90+10,10 x_{1}+30,60 x_{2}+6,30 x_{3}-11,20 x_{1}^{2}+11,30 x_{1} x_{2} \\
\hat{y}_{5}=139,29+4,63 x_{1}+19,75 x_{2}+16,13 x_{3}-5,41 x_{1}^{2}+7,00 x_{1} x_{2} \\
\hat{y}_{6}=146,86+4,87 x_{1}+15,62 x_{2}+27,00 x_{3}-3,98 x_{1}^{2}+4,75 x_{1} x_{2}
\end{gathered}
$$

Os alvos para a otimização das respostas são apresentados em 28.

$$
T_{1}=190 ; T_{2}=185 ; T_{3}=185 ; T_{4}=190 ; T_{5}=185 ; T_{6}=185
$$

As restrições para este problema estão descritas em 29.

$$
\begin{aligned}
& -1,000 \leq x_{1} \leq 1,000 ;-1,000 \leq x_{2} \leq 1,000 ;-1,000 \leq x_{3} \leq 1,000 ; 185 \leq \hat{y}_{1} \leq 195 ; \\
& 170 \leq \hat{y}_{2} \leq 195 ; 170 \leq \hat{y}_{3} \leq 195 ; 185 \leq \hat{y}_{4} \leq 195 ; 170 \leq \hat{y}_{5} \leq 195 ; 170 \leq \hat{y}_{6} \leq 195
\end{aligned}
$$

A função objetivo, resultante da aglutinação das respostas para este estudo de caso está descrita em 30 . 
$D P M=\frac{\left|\frac{\hat{y}_{1}-190}{190}\right|+\left|\frac{\hat{y}_{2}-185}{185}\right|+\left|\frac{\hat{y}_{3}-185}{185}\right|+\left|\frac{\hat{y}_{4}-190}{190}\right|+\left|\frac{\hat{y}_{5}-185}{185}\right|+\left|\frac{\hat{y}_{6}-185}{185}\right|}{6} \cdot 100$

\subsection{Formas de Aplicação do AG e do GRG}

Nesta seção é apresentada a forma de implementação das técnicas utilizadas (AG e GRG).

\subsubsection{Algoritmo Genético (AG)}

A implementação do AG, apresentada no Quadro 2, foi desenvolvida no software Scilab v. 5.5.2. O Scilab é um software gratuito disponibilizado pela Scilab Enterprises e conta com a função optim_ga que consiste em uma sub-rotina que facilita a implementação do AG.

Dependendo dos valores utilizados nos parâmetros do $A G$, o resultado obtido pode ser o valor desejado (ótimo global), um valor próximo ao desejado (ótimo local) ou ainda um valor muito diferente.

A fim de padronizar os parâmetros do $A G$ foram realizados um total de 10 testes utilizando o Caso 1 descrito na seção 3.1.1. Foram então determinados os melhores valores para os parâmetros específicos do $A G$ em função do resultado obtido e do custo computacional envolvido. 
Quadro 2 - Representação do método Algoritmo Genético utilizado para a melhoria de processos

Algoritmo Genético

$N \leftarrow$ Número de gerações

$i \leftarrow 1$

$P \leftarrow$ Tamanho da população

$P c \leftarrow$ Probabilidade de Cruzamento

$P m \leftarrow$ Probabilidade de Mutação

$C \leftarrow$ Probabilidade de cruzamento dos piores indivíduos

$D \leftarrow$ incremento utilizado na criação da população

Min $\leftarrow$ Valor mínimo das variáveis de decisão

Max $\leftarrow$ Valor máximo das variáveis de decisão

Gere aleatoriamente uma população inicial $x$

enquanto $(N>I)$ faça

Ranqueie a população de acordo com a aptidão

Sorteie membros da população para cruzamento ou eliminação em função do resultado do ranking

enquanto $(P>j)$ faça

Sorteie dois membros da população pré-selecionada

se ( $P C>$ número aleatório) então

Realize cruzamento com um ponto de corte entre sorteados

senão

Mantêm os cromossomos inalterados na próxima geração

fim-se

se $(P m>$ número aleatório) então

Realize mutação no cromossomo

fim-se

fim-enquanto

Imprime $x$

fim-algoritmo

\subsubsection{Gradiente Reduzido Generalizado (GRG)}

O método GRG presente no software Microsoft Excel® versão 2013 por meio do aplicativo Solver foi empregado na obtenção da solução dos problemas.

Este aplicativo conta com o GRG2 (segunda geração) já contendo melhorias no algoritmo o tornando mais rápido e eficiente.

Para a aplicação deste método foram necessárias três etapas:

a) Implementação da Função Objetivo: foi obtida respeitando os alvos de cada estudo de caso associada ao método de aglutinação DPM;

b) Variáveis de Decisão: os valores iniciais ficaram definidos como sendo iguais a zero, a fim de se garantir o mesmo início para todas as situações. São as 
variáveis a serem otimizadas a fim de encontrar o melhor arranjo experimental;

c) Especificação das Restrições: foram implementadas de forma a restringir o algoritmo a permanecer dentro da região viável de busca. As restrições foram obtidas de acordo com as particularidades de cada caso especificado.

Após o estabelecimento destes parâmetros foi escolhida a utilização do GRG como o algoritmo de busca a ser utilizado pelo Solver e selecionada a opção de minimizar a função objetivo, que nesse trabalho consiste na aglutinação da DPM.

\section{RESULTADOS E DISCUSSÃO}

A seguir são apresentados os resultados obtidos na calibração dos parâmetros da meta-heurística $A G$ e na busca pelas melhores condições de processo dos estudos de caso.

\subsection{Calibração dos Parâmetros da Meta-Heurística Algoritmo Genético}

O primeiro parâmetro calibrado da meta-heurística $A G$ foi a quantidade de gerações que é o que mais influencia no custo computacional deste algoritmo.

A quantidade de gerações foi variada entre 50 a 500 gerações, e o resultado pode ser observado na Figura 7.

Figura 7 - Gráficos correlacionando a DPM (eixo das ordenadas) com o número de gerações (eixo das abscissas) em função do número total de gerações realizadas

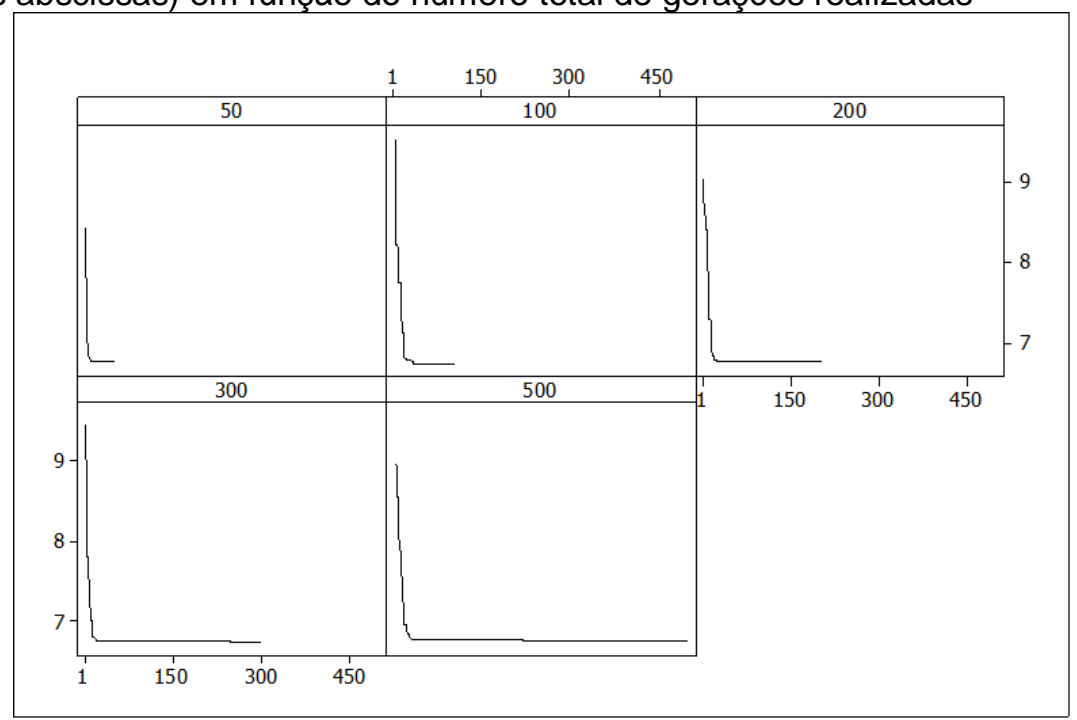

Revista Produção Online. Florianópolis, SC, v.17, n. 2, p. 592-619, 2017. 
Os resultados apresentados na Figura 7 indicam que todos os valores utilizados tendem a convergir para o mesmo valor de DPM, porém, com a utilização de um menor número de gerações, é possível atingir este ponto com um custo computacional menor. Para garantir uma boa convergência em uma gama maior de problemas diferentes sem sacrificar o custo computacional optou-se por utilizar um tamanho de população igual a 100.

O segundo parâmetro foi o tamanho da população. Os resultados obtidos são apresentados na Figura 8.

Figura 8 - Gráficos correlacionando a DPM (eixo das ordenadas) com o número de gerações (eixo das abscissas) em função do tamanho da população

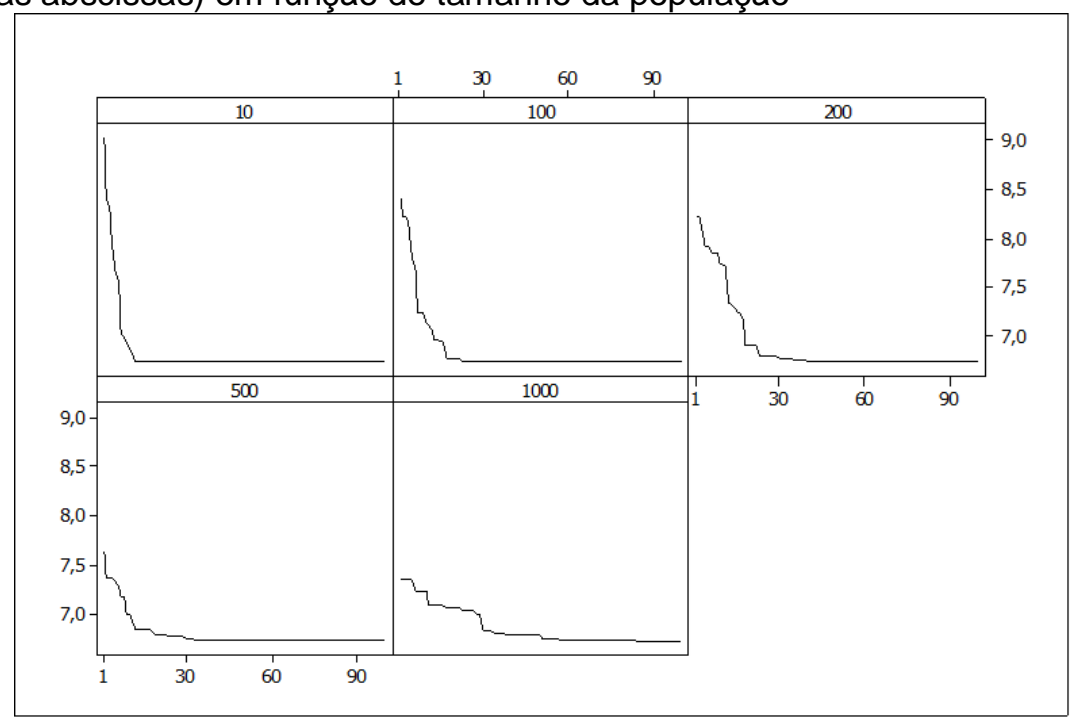

Na Figura 8 pode-se observar que quanto maior o tamanho da população, menor é a DPM alcançada na primeira geração, entretanto, todos os valores utilizados atingem um valor próximo na última geração, e como o tamanho da população também é um fator que incide diretamente no custo computacional do algoritmo, optou-se em adotar o tamanho da população igual a 100 a fim de equilibrar o desempenho do algoritmo com seu custo computacional.

O terceiro parâmetro calibrado foi o número de cruzamentos efetuados. Os resultados estão apresentados na Figura 9. 
Figura 9 - Gráficos correlacionando a DPM (eixo das ordenadas) com o número de gerações (eixo das abscissas) em função do número de cruzamentos

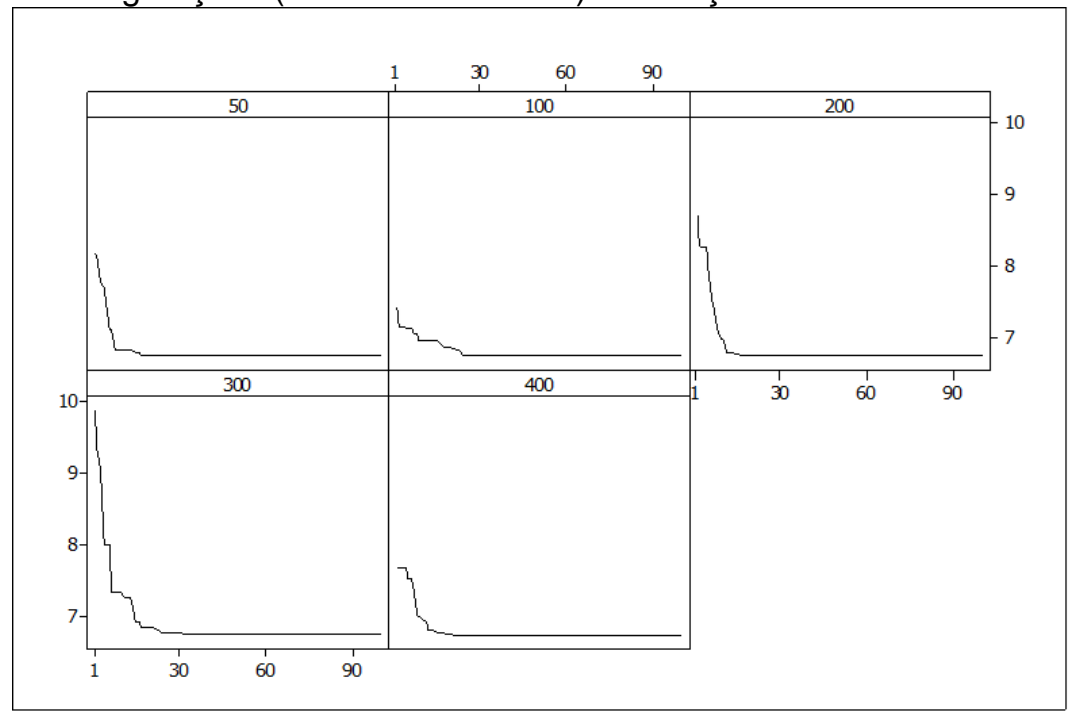

Como mostrado na Figura 9, todos os valores testados convergem para a mesma resposta, portanto, optou-se neste parâmetro pela escolha do valor 100 por ser o que aparentemente tem uma convergência mais rápida.

O quarto parâmetro calibrado foi a probabilidade de mutação, variada em uma faixa entre 0,15 e 0,75. Os resultados são apresentados na Figura 10.

Figura 10 - Gráficos correlacionando a DPM (eixo das ordenadas) com o número de gerações (eixo das abscissas) em função da probabilidade de mutação

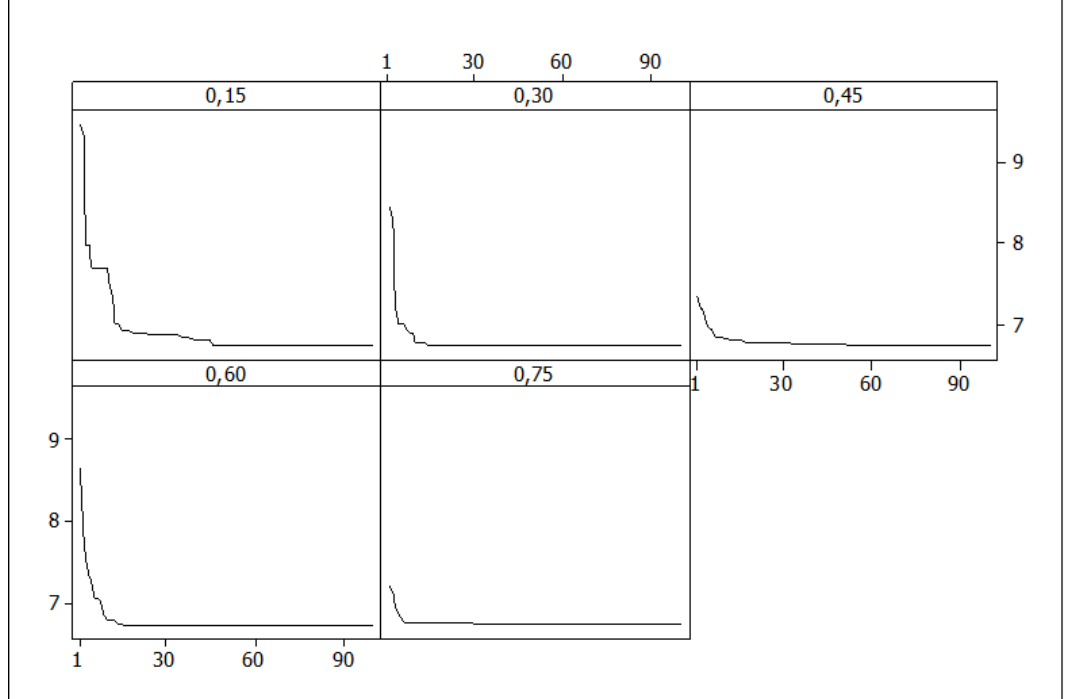

A Figura 10 indica que todas as probabilidades de mutação testadas convergem para a mesma região de resposta, portanto o valor escolhido a ser utilizado neste trabalho foi 0,45 , pois entre todos os valores testados foi o que 
demonstrou uma convergência mais rápida.

Em função dos resultados obtidos, os parâmetros adotados para a Meta-heurística AG, em todos os testes realizados neste estudo, encontram-se sumarizados na Tabela 1.

\begin{tabular}{cc} 
Tabela 1 - Parâmetros calibrados para a Meta-heurística Algoritmo Genético \\
\hline Parâmetro & Valor adotado \\
\hline Número de Gerações & 100 \\
Tamanho da População & 100 \\
Número de Cruzamentos & 100 \\
Probabilidade de Mutação & 0,45 \\
\hline
\end{tabular}

\subsection{Busca pelas Melhores Condições de Processo dos Estudos de Caso}

Os resultados obtidos na determinação das condições que acarretam em uma melhoria dos processos apresentados nos estudos de caso estão sumarizados na Tabela 2.

Tabela 2 - Resultados da busca pelas melhores condições dos processos para a comparação entre os métodos GRG e AG

\begin{tabular}{ccccccccc}
\hline \multirow{2}{*}{ Variáveis } & \multicolumn{2}{c}{ Estudo de Caso 1 } & \multicolumn{2}{c}{ Estudo de Caso 2 } & \multicolumn{2}{c}{ Estudo de Caso 3 } & \multicolumn{2}{c}{ Estudo de Caso 4 } \\
& GRG & AG & GRG & AG & GRG & AG & GRG & AG \\
\hline$x_{1}$ & $-0,305$ & $-0,215$ & $-0,516$ & $-0,516$ & $-0,388$ & $-0,391$ & $-0,085$ & 1,000 \\
$x_{2}$ & 0,866 & 0,055 & $-0,366$ & $-0,366$ & 1,668 & 1,668 & 1,000 & 0,864 \\
$x_{3}$ & $-1,130$ & $-1,049$ & ---- & --- & $-0,484$ & $-0,477$ & 0,865 & 0,791 \\
$\hat{y}_{1}$ & 125,68 & 124,28 & 2,02 & 2,02 & 95,30 & 95,30 & 185,00 & 185,00 \\
$\hat{y}_{2}$ & 1300,00 & 1299,91 & 0,66 & 0,66 & 57,49 & 57,50 & 173,73 & 176,34 \\
$\hat{y}_{3}$ & 500,00 & 499,76 & 1,87 & 1,87 & --- & --- & 172,01 & 176,24 \\
$\hat{y}_{4}$ & 71,74 & 67,53 & 0,37 & 0,37 & --- & --- & 189,05 & 195,00 \\
$\hat{y}_{5}$ & ----- & ----- & ---- & ---- & --- & --- & 171,97 & 174,40 \\
$\hat{y}_{6}$ & ---- & ---- & --- & --- & --- & -- & 185,00 & 186,72 \\
\hline $\boldsymbol{D P M}$ & $\mathbf{0}, 08$ & 6,75 & 19,48 & 19,48 & 2,35 & 2,35 & 4,15 & 3,56
\end{tabular}

Os resultados demonstrados na Tabela 2 indicam que para os estudos de casos 2 e 3, ambos os métodos utilizados neste trabalho ( $A G$ e GRG), convergiram para a mesma região resultando em um ajuste de processo praticamente idêntico, culminando num DPM igual nos dois casos, demonstrando que o método de busca utilizado não influencia no processo de otimização.

Ao analisar os resultados obtidos para a otimização do estudo de caso 1 e o estudo de caso 4 , observa-se que os métodos de busca obtiveram ajustes diferentes do processo, indicando a presença de ótimos locais que podem dificultar a determinação do melhor ajuste do processo, favorecendo portando a implementação do AG como mecanismo mais eficiente para a determinação de um ponto ótimo mais 
robusto. Contudo, deve-se ressaltar que apesar da determinação de um ajuste diferente no processo, os valores obtidos para o DPM não apresentam variações superiores a $3 \%$ (Figura 11).

Figura 11 - Valores de DPM obtidos no processo de otimização

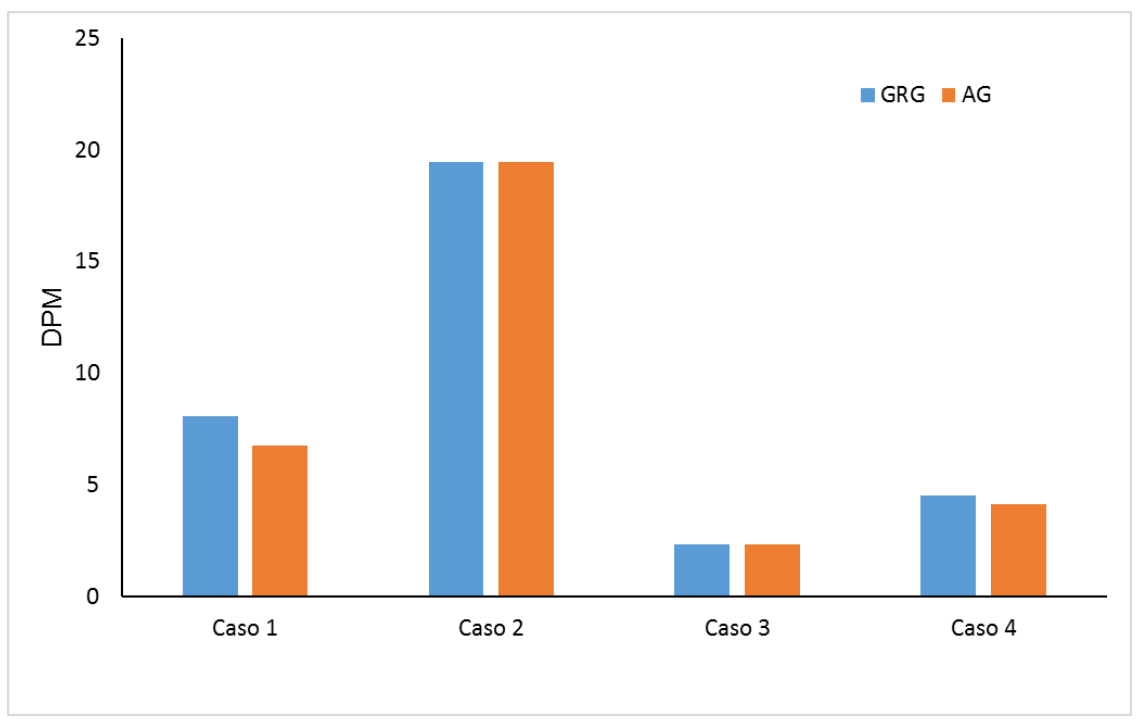

A Tabela 3 apresenta os valores de desvio-padrão e coeficiente de variação calculados em função das dez repetições realizadas para a otimização empregando o AG.

Tabela 3 - Medidas estatísticas de variação das respostas em DPM

\begin{tabular}{ccc}
\hline Caso & Desvio-Padrão & Coeficiente de Variação (\%) \\
\hline 1 & 0,012 & 0,18 \\
2 & 0,000 & 0,00 \\
3 & $9,9 \times 10^{-5}$ & $4,2 \times 10^{-3}$ \\
4 & 0,090 & 2,503 \\
\hline
\end{tabular}

Pode-se averiguar na Tabela 3 que, apesar de ser um método estocástico, o AG apresenta baixa variabilidade nos estudos de caso realizados nesta pesquisa.

A implementação do AG como mecanismo de busca foi efetuada em todos os estudos de caso abordados neste trabalho, utilizando os mesmos parâmetros de calibração. Em função dos resultados obtidos, é possível se afirmar que a calibração não consistiu de uma etapa limitadora do desempenho da meta-heurística nestes casos. Tal fato, contribui ainda mais para a simplificação da implementação deste método de busca na procura de parâmetros robustos na otimização de processos. 
Os tempos computacionais gastos na utilização do AG foram, em média, 1,35 minutos acima do tempo gasto pelo GRG. Apesar desta diferença, o esforço computacional empregado pelo AG não aparenta ser um fator de inibição a sua utilização quando comparado ao GRG.

\section{CONCLUSÕES}

A aplicação da meta-heurística AG como mecanismo de busca para otimização de processos com múltiplas respostas demonstrou ser eficiente quando comparado com o desempenho apresentado pelo GRG que é o método atualmente mais utilizado na prática.

A grande vantagem da utilização do AG é a sua capacidade de escapar de ótimos locais, que estão presentes em alguns modelos de otimização de processos com múltiplas respostas. As desvantagens ficam por conta de um maior tempo computacional gasto, e na dificuldade de implementação quando comparado ao GRG.

O fato da obtenção de um ajuste com maior precisão pode justificar a utilização do AG, apesar das desvantagens presentes neste método.

\section{REFERÊNCIAS}

ABADIE, J.; CARPENTIER, J. Generalization of the Wolfe reduced gradient method to the case of nonlinear constraints. In: Optimization, ed. R. Fletcher, Londres: Academic Press, 1969.

AVILA, S. L., Otimização multiobjetivo e análise de sensibilidade para concepção de dispositivos. 2006. 148 f. Tese (Doutorado em Engenharia Elétrica) - Universidade Federal de Santa Catarina. Florianópolis, 2006.

BAZGAN, C.; JAMAIN, F.; VANDERPOOTEN, D. Approximate Pareto sets of minimal size for multi-objective optimization problems. Operations Research Letters, v. 43, n. 1, p. 1-6, 2015. http://dx.doi.org/10.1016/j.orl.2014.10.003

CASTILLO, E.; MONTGOMERY, D.; McCARVILLE, D. Modified desirability functions for multiple response optimization. Journal of Quality Technology, v. 28, p. 337-345, 1996.

CHENG, C. B.; CHENG, C. J.; LEE, E. S. Neuro-fuzzy and genetic algorithm in multiple response optimization, Computer and Mathematics with Applications, v. 44, n. 12, p. 1503-1514, 2002. http://dx.doi.org/10.1016/S0898-1221(02)00274-2

DERRINGER, G.; SUICH, R. Simultaneous optimization of several response variables. Journal of Quality Technology, v. 12, n. 4, p. 214-219, 1980. 
DEHURI, S.; CHO, S.B. Multi-criterion Pareto based particle swarm optimized polynomial neural network for classification: A review and state-of-the-art. Computer Science Review, v.3, p. 19-40, 2009. http://dx.doi.org/10.1016/..cosrev.2008.11.002

DÍAS-GARCÍA, J.A.; BASHIRI, M. Multiple response optimization: An approach from multiobjective stochastic programming. Applied Mathematical Modelling, v. 38, n. 7-8, p. 2015-2027, 2014. http://dx.doi.org/10.1016/j.apm.2013.10.010

FAGHIHI, V.; REINSCHMIDT, K.F.; KANG, J.H., Construction scheduling using genetic algorithm based on building information model, Expert Systems with Applications, v. 41, n. 16, p. 7565-7578, 2014.

http://dx.doi.org/10.1016/j.eswa.2014.05.047

FOGEL, L. J.; OWENS, A. J.; WALSH, M. J. Artificial intelligence through simulated evolution. New York: John Wiley, 1966.

GOLDBERG, D. E. Genetic algorithms, In Search: optimization and machine learning. Berkeley: Addison-Wesley, 1989.

GOMES, F.M. ; PEREIRA, F.M. ; SILVA, M.B. ; MARINS, F.A S. . Aplicação da Metaheuristica Algoritmo Genético na Otimização de Problemas com Múltiplas Respostas. In: Encontro Nacional de Engenharia de Produção ENEGEP, 2015, Fortaleza. Anais do Encontro Nacional de Engenharia de Produção ENEGEP e ICIEOM, 2015.

HARIDY, S., GOUDA, S. A., WU, Z. An integrated framework of statistical process control and design of experiments for optimizing wire electrochemical turning process. International Journal of Advanced Manufacturing Technology, v.53, p. 191-207, 2011. http://dx.doi.org/10.1007/s00170-010-2828-7

HAMMOUCHE, K.; DIAF, M.; SIARRY, P. A Comparative study of various meta-heuristic techniques applied to the multilevel thresholding problem. Engineering Applications of Artificial Intelligence, v. 23, p. 676-688, 2010.

http://dx.doi.org/10.1016/j.engappai.2009.09.011

HOLLAND, J. H. Adaptation in natural and artificial systems. Michigan: University of Michigan Press, 1975.

IGNÍZIO, J.P., CAVALIER, T.M. Linear Programming. Englewood Cliffs: Prentice Hall, 1994.

KHURI A.; CONLON M. Simultaneous optimization of multiple responses represented by polynomial regression functions. Technometrics, v. 23, n. 4, p. 363-375, 1981.

http://dx.doi.org/10.2307/1268226

KHURI, A.I.; CORNELL, J.A. Response Surfaces: Designs and Analyses. New York: Marcel Dekker Inc., 1987.

KIM, K.J.; LIN, D.K.J. Optimization of multiple responses considering both location and dispersion effects. European Journal of Operational Research, v. 169, p. 133-145, 2006. http://dx.doi.org/10.1016/j.ejor.2004.06.020 
KÖKSOY, O.; YALCINOZ, T. Mean square error criteria to multiresponse process optimization by a new genetic algorithm. Applied Mathematics and Computation, v. 175, n. 2, p. 1657-1674, 2006. http://dx.doi.org/10.1016/j.amc.2005.09.011

LASDON, L. S.; WAREN, A. D.; RATNER, M. W. GRG2 Users's Guide University ofTexas at Austin, 1980.

MELO, A.; CATEN, C.S.T.; SANT'ANNA, A.M.O. Otimização dos parâmetros de usinagem na manufatura do ferro fundido. Revista Produção Online, v.13, n. 1, p. 375-388, 2013. http://dx.doi.org/10.14488/1676-1901.v13i1.1200.

MENDES, J. M. A comparative study of crossover operators for genetic algorithms to solve the job shop scheduling problem. Wseas Transactions on Computers, v. 12, n. 4, p. 164173, 2013.

MONTGOMERY, C.D.; RUNGER, G.C. Estatística aplicada e probabilidade para engenheiros, 5 Ed., LTC, 2012.

PAULING; L. The Nature of the Chemical Bond. Ithaca: Cornell University Press, 1960.

RECHENBERG, I. Evolutionsstrategie: Optimierung technischer systeme nach prinzipien der biologischen evolution, Stuttgart: Frommann-Holzboog, 1973.

REEVES, C. Genetic algorithms. In: Handbook of Metaheuristics. New York: Springer, 2003.

ROSEN, J. B. The gradient projection method for nonlinear programming. Part I: Linear constraints. Journal of the Society for Industrial and Applied Mathematics, v. 8, n. 1, p. 181-217, 1960.

SUDENG, S.; WATTANAPONGSAKORN, N. Post Pareto-optimal pruning algorithm for multiple objective optimization using specific extended angle dominance. Engineering Applications of Artificial Intelligence, v. 38, p. 221-236, 2015. http://dx.doi.org/10.1016/j.engappai.2014.10.020

TORRES JÚNIOR, N.; QUININO, R. C. O jogo da catapulta para compreender o planejamento e análise de experimentos: proposta de uma abordagem lúdica de ensino. Revista Produção Online, v. 14, n. 3, p. 939-971, 2014. http://dx.doi.org/10.14488/1676-1901.v14i3.1576

TSAI, C.; TONG, L.; WANG, C. Optimization of Multiple Responses Using Data Envelopment Analysis and Response Surface Methodology. Tamkang Journal of Science and Engineering, v. 13, n. 2, p. 197-203, 2010.

VELDHUIZEN, D.A.V.; LAMONT, G.B. Multiobjective evolutionary algorithms: analyzing the state-of-the-Art. In: Evolutionary Computation, Cambridge: MIT Press, v. 8, n. 2, p. 125$147,2000$.

VINING G. A compromise approach to multiresponse optimization. Journal of Quality Technology, v. 30, n. 4, p. 309-313, 1998. 
WAREN, A. D.; LASDON, L. S. The status of nonlinear programming software. Operations Research, v. 27, n. 3, p. 431-56, 1979.

WEISE, T., Global Optimization Algorithms - Theory and Application, $2^{\mathrm{a}}$ ed, (2009). Disponível em: http://www.it-weise.de/projects/book.pdf=> acessado em 18 mar. 2015.

WOLFE, P. The Reduced Gradient Method. In: Recent Advances in Mathematical Programming. New York: R. L. Graves and P. Wolfe, 1963.

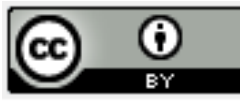

Artigo recebido em 27/08/2016 e aceito para publicação em 12/05/2017

DOI: http://dx.doi.org/10.14488/1676-1901.v17i2.2566 